\title{
Behavioral Cardiovascular Risk Factors: Changing Perspective to Approach the Problem
}

\author{
Giuseppe Gullace* \\ ${ }^{1}$ Prince Sultan Cardiac Center, Qassim \& Genetic laboratory at Qassim University, Kingdom of Saudi Arabia \\ ${ }^{2}$ Multimedica Hospital Group, Milan, Italy
}

Submission: October 25, 2016; Published: November 09, 2016

*Corresponding author: Giuseppe Gullace, Consultant Cardiologist, Multimedica Hospital Group, Via San Leonardo 1123864 Malgrate (Lecco), Italy, Tel: +39 3204337560; Email: ggullace47@gmail.com; j.wallace@alice.it

\begin{abstract}
Several studies indicate that psychological factors strongly influence the neuro hormonal and behavioral cardiovascular risk factors and the course of coronary artery disease and event onset. New epidemiologic evidence for the association between psychosocial risk factors and coronary artery disease identifies pathologic mechanisms that may be responsible for this association. Not much attention is paid to finding and managing the stressing factors that provoke psychological distress and then multiple adverse peripheral effects. This short review highlights a new way to look at the behavioral risk factors; putting attention to what there is behind the onset of stress and psychological distress that are related with cardiovascular risk factors, atherosclerosis and events. The role of cardiologist in finding out, assessing and managing stressors and behavioral risk factors is emphasized and an emerging cardiological field based on the understanding that psycho behavioral risk factors require a new approach and a new more effective health care system is suggested.
\end{abstract}

Keywords: Atherosclerosis; Behavioral risk factors; Neuro hormonal and Psycho behavioral structures; Stress

\section{Introduction}

Atherosclerosis is a multi factorial disease of polygenic inheritance and the interrelation with environmental and social factors adds to complexity of the disease; in addition behavioral risk factors contribute to atherosclerosis occurrence and development. The relationship between risk factors and cardiovascular and cerebrovascular diseases has been widely elucidated, including their definition in unmodifiable (such as age, sex, familiarity, inheritance), modifiable/curable (such as obesity, hypertension, physical inactivity, dyslipidemia, diabetes) and psychosocial (such as stress, depression, anxiety, type A personality, hostility and anger) [1-7]. The use of traditional factors for risk stratification is well established and those patients identified are at an increased risk. The longterm follow-up of the Framingham Offspring cohort [8] allowed the investigators to derive 30-year risk equations for hard and overall cardiovascular event. However, depending on this traditional risk factors, to assess individual risk might be late and, moreover, there are difficulties to control it.

The European action on secondary prevention through intervention to reduce events (EUROASPIRE) survey carried out in eight European countries [9] reports that despite improvements in lipid control, no significant changes in blood pressure levels or smoking were obtained and increases in obesity and diabetes were seen in patients with established coronary heart disease. On the other hand, traditional risk factors for the development of atherosclerotic disease, which accelerate the process over time and increase the risk of having a heart attack, stroke or other arterial vascular problem are not present in $50 \%$ of those who develop atherosclerotic problems [10]. These individuals appear to have other genetic disorders or abnormal blood clotting, chronic inflammation of arteries or as yet unknown problems.

Moreover, epidemiological research suggests that psychological factors, stress and undesirable social circumstances play an important role in the occurrence of various cardiovascular diseases [11,12] and are considered major risk factors for coronary heart disease. The coronaryprone behavior pattern, called type A, that was originally described by Friedman \& Rosenman [13] in the late 1950's, has been considered a clinically derived behavioral syndrome characterized by competitive drive, time urgency, hostility, and strong job involvement. Characteristics as anger or aggression of a group of patients have been demonstrated to be important 
factors for cardiovascular and cerebrovascular disease [14] and to be related to carotid atherosclerosis, while depression was associated with endothelial dysfunction in diabetic patients [15], predicts incident coronary artery disease and worsens its prognosis [16-19], and anxiety is considered an independent risk factor for incident coronary artery disease, for cardiac mortality following acute myocardial infarction and cardiac events $[20,21]$. In addition, there are different lifestyle behaviors considered risk factors for coronary artery disease, as they promote atherosclerosis and cardiac events, including unhealthy diet, physical inactivity, smoking, obesity and sexual activity that are related to emotional status and experience of chronic stress [22].

\section{Purpose}

As reported above, the relationship among cardiovascular risk factors, atherosclerosis and cardio-cerebrovascular disease occurrence appears a complex and intriguing issue not yet completely and fully disentangled. The relationship between psychological (stress, anxiety, depression, etc.) and behavioral (over or unhealthy eating, obesity, smoke, physical inactivity, sexual activity reduction/ lack and related diseases such as hypertension, diabetes and dyslipidemia) factors is one of the interesting issues that we try to deal with in the present paper changing perspective to approach the several aspects and to look at them from a different point of view.

\section{The Current Way}

We are convinced, from a wide literature and daily practice, that the four pillars of good health, related to the individual's lifestyle and depending on his behavior, are:
i. No Smoking
ii. Eating little
iii. Walking so much
iv. Sex a lot

What usually occurs today is that smokers are still too many, over and unhealthy eating is widely spread and, consequently, obesity is highly present in general population, only less than $50 \%$ of adult people practice regular physical activity and sexual activity is influenced by several problems such as biological, psychological, social, economic, political, cultural, ethical, legal, historical, religious, and spiritual factors; but also by violence against women, false beliefs and few knowledge's and medical like infections, organic dysfunctions, delivery complications [23]. For the latter, the report concludes: "Sexual and reproductive health is fundamental to the social and economic development of communities and nations, and a key component of an equitable society a vital contribution to making the world a fairer place" [23]. All of them are related to person's psychological factors, behavior and life style.
Within this context, we note that although cardiologists are accustomed to managing lifestyle behaviors such as overeating, obesity, smoking and physical inactivity, they are less likely to assess and treat psychosocial risk factors, perhaps because of their limited time, interest or familiarity with effective strategies and recommendations [24] or because a systematic review, based on few studies, refers that risk assessment, combined with counseling, is associated with favorable but modest changes in patient knowledge and intention to change and in provider prescribing behavior and risk factor control [25].

For instance, regarding physical activity, only $47 \%$ of primary care physicians include an exercise history as part of their initial examination, whereas $13 \%$ of patients report physicians giving advice about exercise and physically active physicians are more likely to discuss exercise with their patients [26-28]. But the main issue of this situation is that the four pillars of good healthy are widely influenced by psychological and behavioral factors in the daily life by modifying the capability to adequately manage the relationship with either the inside self emotions and feelings or the outside, people and events included. More attention should be paid to study all these aspects.

\section{The structure of the individual}

The individual may be summarily defined as a complex organization in which neural, hormonal, cellular, and genetic mechanisms are strictly related to social behaviors whose main task is to help the organism to survive, reproduce, and care for offspring sufficiently long that they too reproduced [25]. This means that neuro hormonal structure of individual is related to psycho behavioral one and that both influence one to each other [29]. There exisists a wide literature reported in an interesting review [30] that lists the numerous examples of reciprocal influence of neuro hormonal and psycho behavioral structures. In an interesting paper Rozanski et al. [24] consider three psychological components that may be central to developing emotional and coping flexibility: Vitality, Emotional Competence and Positive response mechanisms. Vitality reflects the presence of energy and enthusiasm and a sense of aliveness [31]; it is characterized by two positive emotions, joy and interest, it is fueled by both a sense of purpose and a sense of self-worth and connotes a sense of positive excitement [24] and attitude.

Vitality promotes two adaptive responses (which may in turn positively influence vitality itself): the development of various positive response mechanisms (such as patience, discipline, impulse control, strong social support, positive coping skills, optimism) and emotional competence, that represents the ability to regulate emotions across a range of situations [32], important trait termed "emotional flexibility," [33]. Briefly, a strong sense of purpose coupled with a sense of self-worth derive benefit in terms of a greater sense of vitality; this provides energy needed to develop and mantain greater emotional competence and 
positive response mechanisms that in turn provide a stabilizing force for maintaining a sense of vitality [24].

All this process well represents the individual's relationship (balance) between inner and outer and between neuro hormonal and psycho behavioral structures. When several factors negatively affect the structures the relationship is unbalanced and anxiety, depression, stress, affective disorders and psychological instability arise and grow up, evoking central responses that cause multiple peripheral adverse effects such as endothelial dysfunction, insulin resistance, obesity, hypertension, inflammation, platelet activation, somatic symptoms [24]. It is evident that stress as a complex of several psychological situations, is the effect of multiple long acting factors that progressively determine unbalancing of neuro hormonal and psycho behavioral structures reducing the sense of purpose and the sense of self-worth negatively modifying vitality, emotional competence and positive response mechanisms and consequenlty reinforcing cardiovascular risk factors.

\section{Changing perspective to approach the problem}

"When you change the way you look at things, the things you look at will change" is one of the quotes from Wayne W Dryer, psychologist and philosopher [34]. This is very useful to do when the situation and thinking become temporarily intriguing and complex and the solutions to problems are confused and bleary. It is also useful when it is necessary to look at problems from a different point of view in order to find more or better solutions or the best one or when a new way to cope situations and problems is needed to give suitable answers and acceptable explanations to complicated and complex questions even if they concerns acquired and apparently established issues. Concerning the behavioral risk factors and according with that reported above it is important to experiment a new point of view orienting the attention towards person's perception of them self, his behavior and his inner/outer relationship unbalance [35]. This means that it is necessary to take into account that all acquired risk factors are a consequence of a bad relationship that persons have, either with themselves (inner) or with the others (outer), but it is also important to know what there is behind the behavioral unbalancing [35]. We may hypothesize the following process: (Figure 1) where several factors disturb along the time the neuro hormonal and psycho behavioral balance provoking stress; this induces multiple adverse peripheral effects related to behavioral risk factors and then the onset of the disease.

\section{Internal/External Factors
Acting as stressors
Neuro hormonal and Psycho behavioral
Unbalancing
Anxiety, Depression, Anger, Frustration, Unsatisfaction, Lack of Motivation and Purposefulness
Hypothalamic Pituitary Adrenal axis - Sympathetic Nervous System - Behaviors
Evoked central responses
Behavioral Risk Factors
$\mathbf{v}$
Disease}

In the light of this process we should look at problem paying more attention to the persons (subjects or preclinical patients), striving to understand what there is behind them that pushes to unsuitable and deleterious lifestyle. Hippocrates wrote that "it could not be possible to know medical practice for those who do not know who and what is man; this is what must be understood by those who shall take correctly care of men; if common man's comprehension will be missed... also reality will be missed." Investigators and clinical practitioners pay great attention to the disease; a large literature exists considering cardiovascular risk factors and their potential effect on disease onset [35]. However, at the same time, literature is stingy in considering, for example, that smoke, stress, obesity and alimentary disorders are not the cause of something but rather the effect of an incorrect and inappropriate interpretation and management of relationships that persons establish with those.

A different approach to behavioral risk factors should be experimented orienting the attention towards what there is behind their onset. A statement from the World Health Organization (WHO) asserted that: "it is impossible to change social values and health behavior without creating the necessary atmosphere and condition" [36]. The strong and robust relationship between psycho behavioral risk factors and coronary artery disease suggests that cardiologists and physicians in general need to be proactive in addressing this important aspect of patient care [25]. Querying patients about psychosocial risk factors conveys the message that these factors 
are important and relevant to providing optimal care. Screening has been suggested using structured interviews or validated questionnaires. The proposal is to obtain within the medical history and assessment of the patient additional information about psychosocial risk factors through questions regarding emotional factors, such as depression, anxiety, and anger; chronic stressors, such as work strain and home stress; and somatic complaints that maybe stress-related, such as fatigue and disrupted sleep [25].

We think that during the patient assessment questions should be addressed deeply to find out what there is behind stress and anxiety and which factors provoke the psycho behavioral distress; more attention should be paid to the patient's neuro hormonal and psycho behavioral profile to understand better the level of factor stressors, what is disturbing the patient and what is linked to risk factors [35]. Many post myocardial infarction or post heart surgery patients usually come daily to our cardiology offices asking in many different ways, and deeply convinced, to help them to quit smoking, to reduce weight, how they can change their deleterious lifestyle; we cannot dismiss patients with few hasty words. We must take care of their psyco behavioral problems trying to identify and understand the distress stressor factors and helping him to remove them. The role of the cardiologist is important by exploring various ways for effective clinical involvement and also by managing these situations; in particular complicated cases, the specific involvement of other specialists is recommended [37-39].

In our daily experience with patients' problems we use, with positive results in the majority of them, an approach in four phases during a weekly counseling with a single patient. The first phase aims to establish a relationship in empathy; the second phases aims to help the patient to look at behind of things and events, to look for into his past history and tell it; the third phase has the aim to guide patients to realize what of his history is disturbing or not and help him in his decision to remove them; the final phase is used to reinforce patient's resilience in order to help him to find and appropriate use all the capabilities he has put aside. During counseling, information on risk factors, cardiovascular diseases, relationship among neuro hormonal structure and function, behavior and cardiovascular apparatus are given.

The final objective is to help the patient to recognize his own problems, to help him to look at things from a different point of view, to give him the necessary information to better manage his disturbing factors and to help him to recover the emotional energy and the positive sense of purpose and self worth: in few words to reappropriate of himself, improve his lifestyle and look at the future in a better way.

\section{The Way Forward}

Social and economic problems provide the field, the person's perception of themselves regarding their internal world and wishing to be themselves balanced against the external world are the main topics to be stressed in the incoming future, and cardiologists and all the specialists involved, should play a preminent role in promoting more attention, more time to spend and more research for a better comprehension of stress dynamics in behavioral risk factors relationship for an increasing time to dedicate to treatment. Behavioral Cardiology could be an emerging field of medical practice that aims to recognize, manage and treat stressing factors that produce adverse lifestyle behaviors, emotional distress, and chronic life stress contributing to promote atherosclerosis and adverse cardiac events. The future may be achieved talking much more with the patients, trying to assess his psychological profile, helping him in recognizing and managing stressing factors and emotional distress and, recovering the old way of medical profession's beauty to look at the person with the disease and not simply at the disease.

\section{References}

1. Kotseva K, Wood D, De Backer G, De Bacquer D, Pyörälä K, et al (2010) EUROASPIRE III. Management of cardiovascular risk factors in asymptomatic high-risk patients in general practice: cross-sectional survey in 12 European countries. Eur J Cardiovasc Prev Rehabil 17(5): 530-540.

2. Kotseva K, Wood D, De Bacquer D, De Backer G, Rydén L, et al. (2016) EUROASPIRE IV: a European Society of Cardiology survey on the lifestyle, risk factor and therapeutic management of coronary patients from 24 European countries. Eur J Prev Cardiol 23(6): 636-648.

3. DAWBER TR, KANNEL WB, LYELL LP (1963) An approach to longitudinal studies in a community: the Framingham Study. Ann N Y Acad Sci 107: 539-556.

4. Fried LP, Borhani NO, Enright P, Furberg CD, Gardin JM, et al. (1991) The Cardiovascular Health Study: design and rationale. Ann Epidemiol 1(3): 263-276.

5. Kannel WB, Feinleib M, McNamara PM, Garrison RJ, Castelli WP (1979) An investigation of coronary heart disease in families. The Framingham offspring study. Am J Epidemiol 110(3): 281-290.

6. Friedman GD, Cutter GR, Donahue RP, Hughes GH, Hulley SB, et al. (1988) CARDIA: study design, recruitment, and some characteristics of the examined subjects. J Clin Epidemiol 41(11): 1105-1116.

7. The Atherosclerosis Risk in Communities (ARIC) Study: design and objectives. The ARIC investigators. (1989) Am J Epidemiol 129(4): 687-702.

8. Pencina MJ, D’Agostino RB Sr, Larson MG, Massaro JM, Vasan RS (2009) Predicting the 30-year risk of cardiovascular disease: the Framingham heart study. Circulation 119(24): 3078-3084.

9. Kotseva K, Wood D, De Backer G, De Bacquer D, Pyörälä K, et al. (2009) Cardiovascular prevention guidelines in daily practice: a comparison of EUROASPIRE I, II, and III surveys in eight European countries. Lancet 373(9667): 929-940.

10. Superko HR (1996) Lipid Disorders Contributing to Coronary Heart Disease - An Update. Curr Prob Cardiol 21(11): 733-780

11. Jenkins CD (1976) Recent evidence supporting psychologic and social risk factors for coronary disease. N Engl J Med 294(19): 987-994.

12. Jenkins CD (1976) Recent evidence supporting psychologic and social risk factors for coronary disease. N Engi J Med 294(19): 1033-1038. 
13. FRIEDMAN M, ROSENMAN RH (1959) Association of specific overt behaviour pattern with blood and cardiovascular findings. J Am Med Assoc 169(12): 1286-1296.

14. Matsumoto Y, Uyama O, Shimizu S, Michishita H, Mori R, et al. (1993) Do anger and aggression affect carotid atherosclerosis? Stroke 24(7): 983-986.

15. Kheirabadi GR, Toghani F, Kousha M, Hashemi M, Maracy MR, et al (2013) Is there any association of anxiety-depressive symptoms with vascular endothelial function or systemic inflammation? J Res Med Sci 18(11): 979-983.

16. Lichtman JH, Froelicher ES, Blumenthal JA, Carney RM, Doering LV, et al. (2014) Depression as a risk factor for poor prognosis among patientswith acute coronary syndrome: systematic review and recommendations: a scientific statement from the American Heart Association. Circulation 129(12): 1350-1369.

17. Tim S Nawrot, Laura Perez, Nino Künzli, Elke Munters, Benoit Nemery (2011) Public health importance of triggers of myocardial infarction: a comparative risk assessment. Lancet 377(9767): 732-740.

18. Spindler H, Pedersen SS (2005) Posttraumatic stress disorder in the wake of heart disease: prevalence, risk factors, and future research directions. Psychosom Med 67(5): 715-723.

19. Orth-Gomér K, Wamala SP, Horsten M, Schenck-Gustafsson $K$, Schneiderman N, et al. (2000) Marital stress worsens prognosis in women with coronary heartdisease: the Stockholm Female Coronary Risk Study. JAMA 284(23): 3008-3014.

20. Roest AM, Martens EJ, de Jonge P, Denollet J (2010) Anxiety and risk of incident coronary heart disease: a meta-analysis. J Am Coll Cardiol 56(1): 38-46.

21. Roest AM, Martens EJ, Denollet J, de Jonge P (2010) Prognostic association of anxiety post myocardial infarction with mortality and new cardiac events: a meta-analysis. Psychosom Med 72(6): 563-569.

22. Rozanski A, Blumenthal JA, Kaplan J (1999) Impact of psychological factors on the pathogenesis of cardiovascular disease and implications for therapy. Circulation 99(16): 2192-2121.

23. Glasier A, Gülmezoglu AM, Schmid GP, Moreno CG, Van Look PF (2006) Sexual and reproductive health: a matter of life and death. Lancet 368(9547): 1595-1607.

24. Rozanski A, Blumenthal JA, Davidson KW, Saab PG, Kubzansky L (2005) The Epidemiology,Pathophysiology, and Management of Psychosocial Risk Factors in Cardiac Practice. The Emerging Field of Behavioral Cardiology. J Am Coll Cardiol 45(5): 637-651.

25. Sheridan SL, Viera AJ, Krantz MJ, Ice CL, Steinman LE, et al. (2010) The effect of giving global coronary risk information to adults: a systematic review. Arch Intern Med 170(3): 230-239.
26. Eakin EG, Brown WJ, Marshall AL, Mummery K, Larsen E (2004) Physical activity promotion in primary care: bridging the gap between research and practice. Am J Prev Med 27(4): 297-303.

27. Abramson S, Stein J, Schaufele M, Frates E, Rogan S (2000) Personal Exercise Habits and Counseling Practices of Primary Care Physicians: A National Survey. Clin J Sport Med 10(1): 40-48.

28. Walsh JM, Swangard DM, Davis T, Mc Phee SJ (1999) Exercise counseling by primary care physicians in the era of managed care. Am J Prev Med 16(4): 307-313.

29. Cacioppo JT, Berntson GG, Decety J (2010) Social neuroscience and its relationship to social psychology. Soc Cogn 28(6): 675-685.

30. Marta C Soares, Redouan Bshary, Leonida Fusani, Wolfgang Goymann, Michaela Hau, et al. (2010) Hormonal mechanisms of cooperative Behavior. Phil Trans R Soc B 365: 2737-2750.

31. Ryan RM, Frederick C (1997) On energy, personality, and health: subjective vitality as a dynamic reflection of well-being. J Pers 65(3): 529-565.

32. Gross JJ (1998) Antecedent- and response-focused emotion regulation: divergent consequences for experience, expression, and physiology. J Pers Social Psychol 74(1): 224 -237.

33. Bonanno GA, Papa A, Lalande K, Westphal M, Coifman K (2004) The importance of being flexible: the ability to enhance and suppress emotional expression predicts long-term adjustment. Psychol Sci 15(7): 482-487.

34.https://www.goodreads.com/author/quotes/2960. Wayne_W_Dyer

35. Giuseppe Gullace, Hassan Khalaf (2011) Preclinical Diagnosis and Risk Assessment of Atherosclerosis - At Birth? At Event? When is Best? European Cardiology 7(3): 162-164.

36. WHO (1984) EURO report and studies 98. Anacapri, Italy.

37. Whooley MA, Simon GE (2000) Managing depression in medical outpatients. N Engl J Med 343(26): 1942-1950.

38. Joos SK, Hickam DH (1990) How health professionals influence health behavior: patient provider interaction and health care outcomes. In: Glanz K, Lewis FM, Rimer BK (Eds.), Health Behavior and Health Education: Theory Research and Practice, Jossey-Bass, San Fransisco, CA, USA, pp.216-241.

39. Nancy Houston Miller, Martha Hill, Thomas Kottke and Ira S. Ockene (1997) The multilevel compliance challenge: recommendations for a call to action. A statement for healthcare professionals. Circulation 95: 1085-1090.

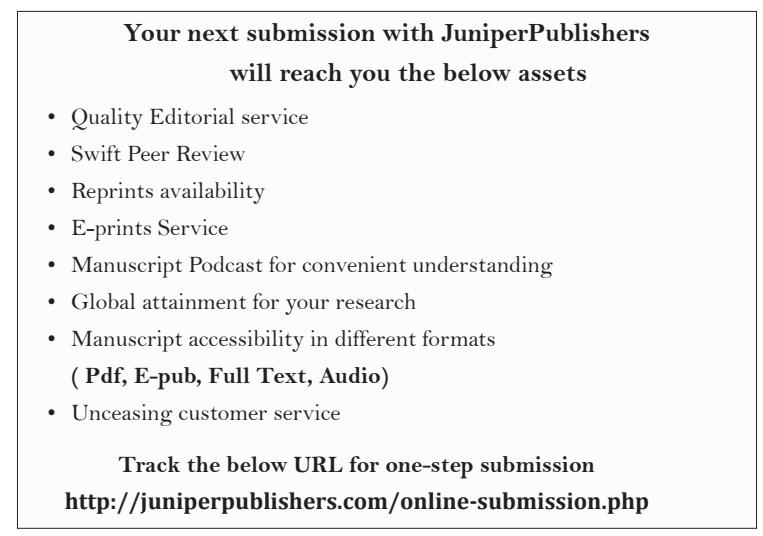

